\title{
Valve in valve implantation of the CoreValve Evolut $R$ in degenerated surgical aortic valves
}

\author{
Marius Schwerg, Karl Stangl, Michael Laule, Verena Stangl, Henryk Dreger
}

Department of Cardiology, Charité - Universitätsmedizin Berlin, Campus Mitte, Berlin, Germany

\begin{abstract}
Background: The new CoreValve Evolut $R$ has an improved design to minimize paravalvular leakage and allows repositioning of the valve. For patients with degenerated bioprosthetic aortic valves, transcatheter aortic valve implantation (TAVI) represents a less invasive option. Herein reported are valve-in-valve (ViV) implantations of this new valve.

Methods: A total of 26 patients (mean age $79.4 \pm 6.1$ years, 17 males and 9 females) were treated for severe prosthesis stenosis $(n=9)$, severe regurgitation $(n=8)$ or severe combination of stenosis and regurgitation $(n=9)$. All patients underwent transthoracic echocardiography before and after ViV implantation.

Results: Valve-in-valve implantation of a CoreValve Evolut $R$ was performed successfully in all patients. The mean transaortic gradient for stenotic valves determined by transthoracic echocardiography was reduced significantly from $37.5 \pm 15.3 \mathrm{mmHg}$ in patients with prosthesis stenosis to $16.3 \pm 8.2$ $\mathrm{mmHg}(p<0.001)$. In all cases with severe prosthesis regurgitation, regurgitation was reduced to none or mild. All-cause mortality after 30 days was $0 \%$.

Conclusions: It was concluded that CoreValve Evolut $R$ is well suited for ViV implantation. (Cardiol J 2018; 25, 3: 301-307)
\end{abstract}

Key words: aortic valve, transcatheter aortic valve implantation, valve-in-valve

\section{Introduction}

For patients with a degenerated bioprosthetic heart valve, transcatheter aortic valve implantation (TAVI) is a less invasive treatment option. A reoperation of these mostly elderly patients with frequent comorbidities is associated with a higher perioperative mortality [1]. Some experience with valve-in-valve (ViV) implantation of the balloon-expandable Edwards Sapien XT valve and the self-expandable Medtronic CoreValve system has already been published [2, 3]. However, data on the new Medtronic CoreValve Evolut R valve is scarce $[4,5]$.

The new Medtronic CoreValve Evolut R has an improved design to minimize paravalvular leakage. In addition, its new delivery system allows for repositioning of the valve [6]. Herein are reported
26 cases of degenerated aortic bioprostheses managed with CoreValve Evolut R.

\section{Methods}

Since 2009, more than 1700 patients underwent TAVI in the documented center. From April 2013 to October 2017, 26 patients were treated for a failing surgical aortic valve with a CoreVave Evolut R. All patients presented with severe comorbidities preferring an interventional approach as determined by an interdisciplinary heart team (Table 1). In all patients, transesophageal echocardiography was performed to determine valve pathology prior to the procedure. The aortic annulus diameter was measured in the mid-esophageal long-axis view and/or by computed tomography. In all cases a transfemoral access was used.

Address for correspondence: Marius Schwerg, MD, Klink für Kardiologie und Angiologie, Charite-Berlin, Berlin, Germany, tel: 004930450613236, fax: 004930450513092, e-mail: marius.schwerg@charite.de

Received: 28.08.2017 Accepted: 01.01.2018 


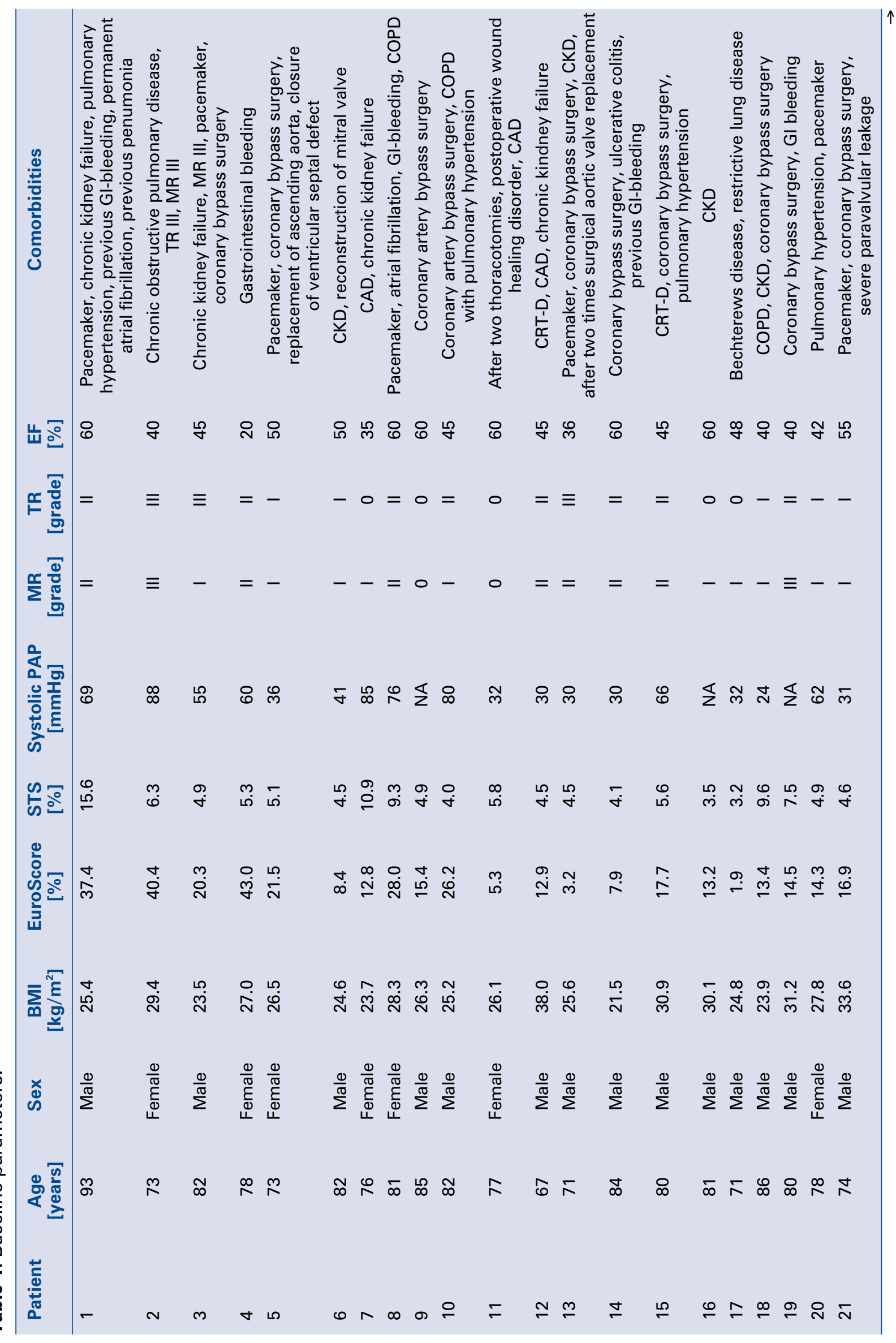




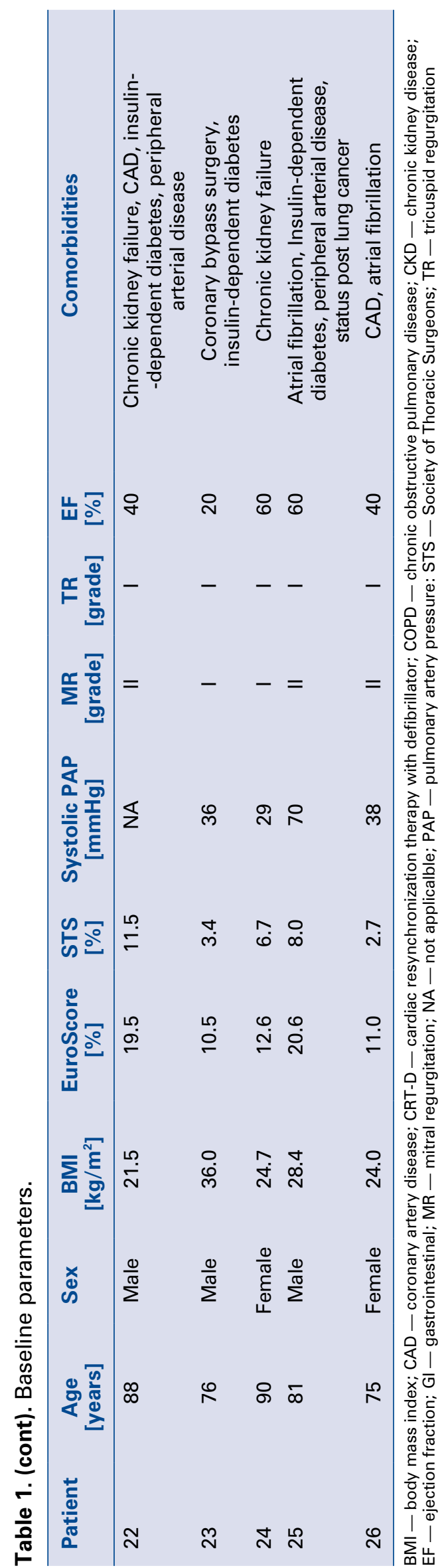

A clinical examination, an electrocardiography and a transthoracic echocardiography were performed before TAVI and before discharge.

TAVI was performed in a specially equipped hybrid suite under general anesthesia by an interdisciplinary heart team consisting of a cardiac surgeon, a cardiologist and an anesthesiologist. All relevant baseline, procedural, and follow-up data were collected retrospectively.

A total of 26 patients (mean age $79.4 \pm 6.1$ years, 17 male and 9 female) were treated for degenerated aortic prosthesis following surgical aortic valve replacement. The exact type of surgical bioprosthesis are listed in Table 2. Severe aortic stenosis was defined as an effective aortic orifice area (EOA) $<1 \mathrm{~cm}^{2} .7$ prostheses showed a high-grade stenosis, 7 prostheses had severe aortic regurgitation and 7 showed high grade stenosis combined with serve aortic regurgitation. The elevated surgical risk is underscored by the STS score for risk of mortality $(6.2 \pm 3.0 \%)$ and EuroScore II $(17.3 \pm 10.5 \%)$. All patients were analyzed for 30 day survival.

\section{Statistical analysis}

Wilcoxon matched pairs signed rank test for statistical significance (GraphPad Prism 6, La Jolla, CA, USA) was performed. A p-value $<0.05$ was considered to be statistically significant. When appropriate, data are presented as box plots with the boundaries of the box as the $75^{\text {th }}$ and $25^{\text {th }}$ percentiles, and with a line in the box indicating the median. Whiskers above and below the box mark maximum and minimum values, respectively.

\section{Compliance with ethical standards}

All procedures performed in studies involving human participants were in accordance with the ethical standards of the institutional and/or national research committee and with the 1964 Helsinki declaration and its later amendments or comparable ethical standards.

\section{Results}

The ViV implantation of the CoreValve Evolut $\mathrm{R}$ was performed successfully in all patients. In 18 patients, an Evolut $23 \mathrm{~mm}$ was used, 5 patients received an Evolut $26 \mathrm{~mm}, 2$ patients received an Evolut $29 \mathrm{~mm}$ and 1 patient received an Evolut $34 \mathrm{~mm}$. The mean transaortic gradient determined by transthoracic echocardiography was reduced significantly from $37.5 \pm 15.3 \mathrm{mmHg}$ in patients with prosthetic stenosis to $16.3 \pm 8.2 \mathrm{mmHg}(\mathrm{p}<0.001)$ 


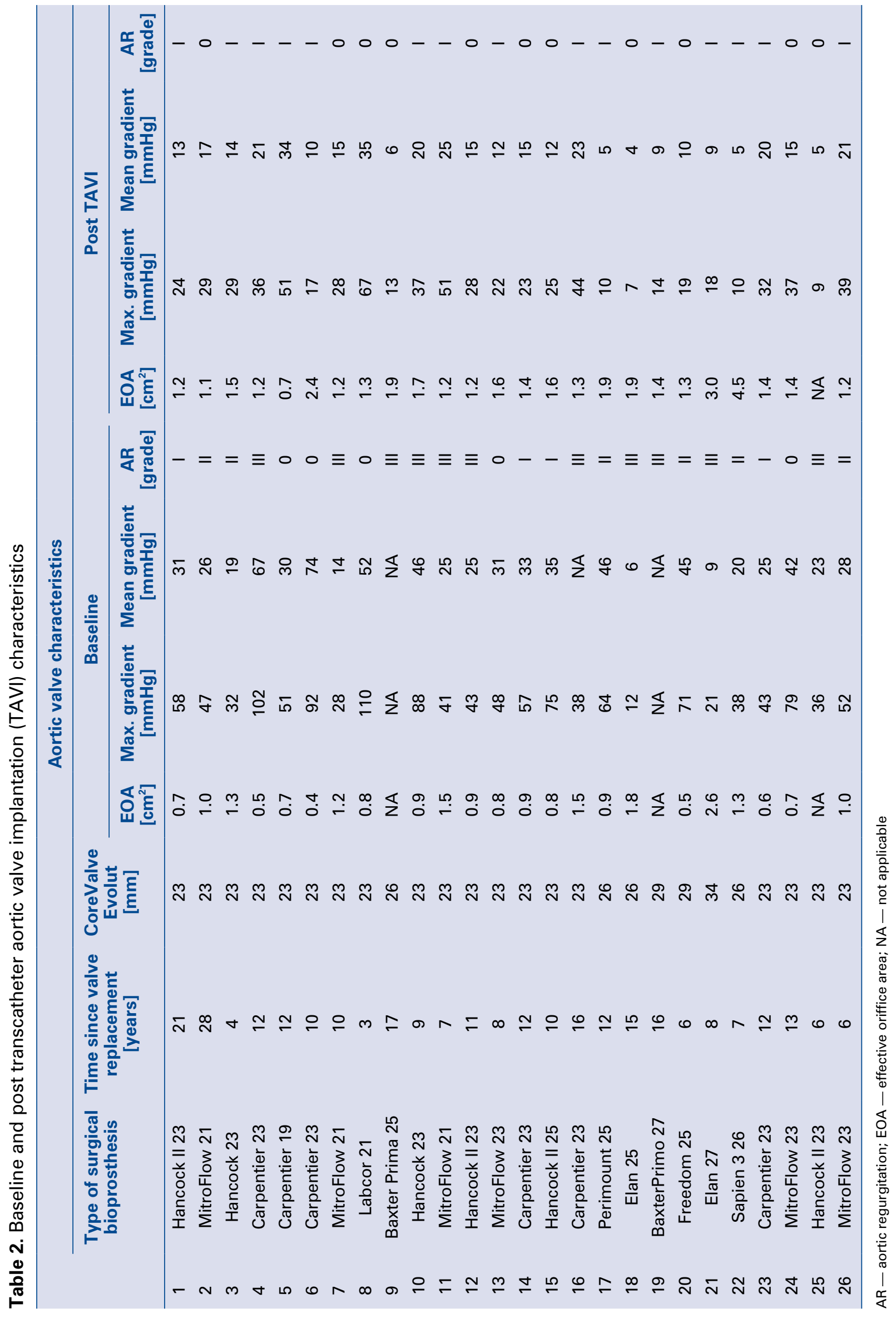




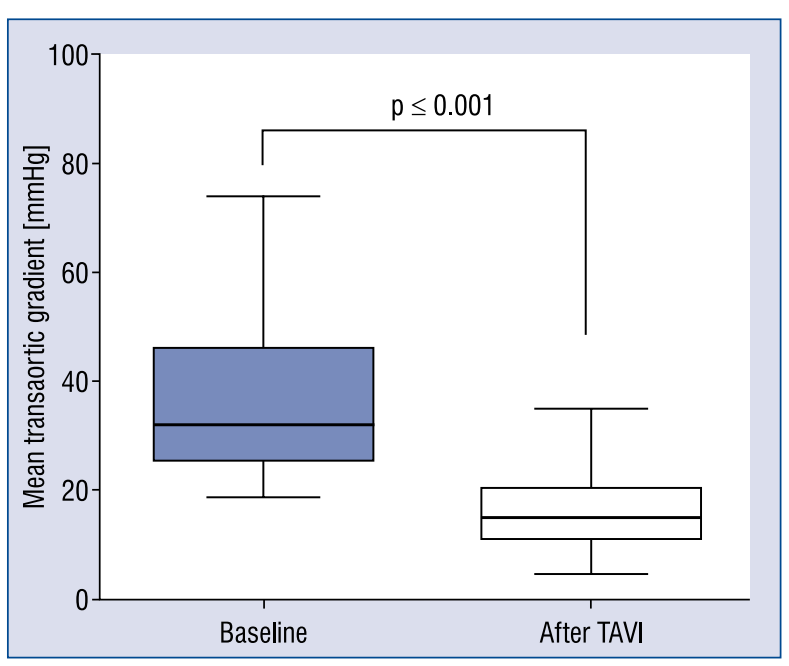

Figure 1. Mean pressure gradients at baseline and after valve-in-valve transcatheter aortic valve implantation (TAVI) $(p<0.001)$.

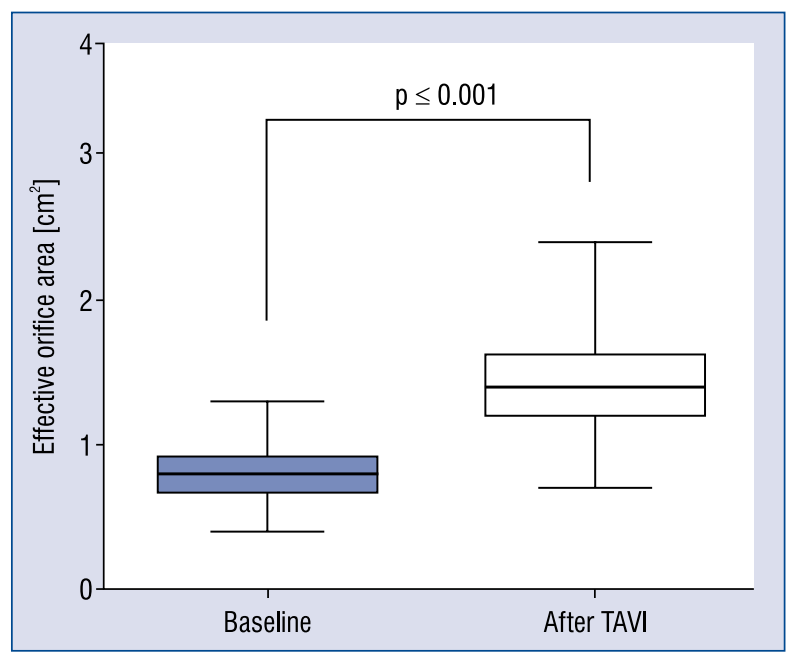

Figure 2. Effective orifice area at baseline and after valve-in-valve transcatheter aortic valve implantation (TAVI) $(p<0.001)$.

(Fig. 1). The EOA increased from $0.8 \pm 0.2 \mathrm{~cm}^{2}$ in patients with prosthesis stenosis to $1.5 \pm 0.4 \mathrm{~cm}^{2}$ $(\mathrm{p}<0.001)$ (Fig. 2). In all cases with severe aortic regurgitation, the regurgitation was reduced to none or mild regurgitation. There was no case of moderate or severe regurgitation after ViV. In 3 patients, a postdilatation was performed due to excessive calcification. The mean EOA was $1.4 \pm$ $\pm 0.4 \mathrm{~cm}^{2}$ for the $23 \mathrm{~mm}$ Evolut valve that was used the most.

In 3 patients, implantation of a permanent pacemaker was necessary. In 2 patients (No. 9 and
No. 21) pacemaker implantation was necessary due to a complete atrioventricular block after TAVI. In these cases, the CoreValve Evolut R had to be implanted deep into the left ventricular outflow tract to avoid obstruction of the right coronary artery for patient 9 and to cover a paravalvular regurgitation in patient No. 21. In 1 patient, the pacemaker was implanted due to symptomatic sick sinus syndrome 6 days after ViV. In all other cases, no new conduction disturbances were observed.

In patient No. 5, the CoreValve Evolut $\mathrm{R}$ dislocated into the ascending aorta after deployment. A second CoreValve Evolut $\mathrm{R}$ was implanted into the surgical valve fixating the first valve in the ascending aorta without obstruction of the brachiocephalic artery. In this patient, a reduction of the mean transaortic gradient was not accomplished.

In 4 patients (No. 7, 11, 16 and 26), the transaortic gradient was not reduced or even increased slightly. In these patients a combination of stenosis and regurgitation was an indication for ViV. The regurgitation was reduced effectively in all patients. In these patients, a short acceleration time of the transaortic outflow $(<100 \mathrm{~ms}$ ) was measured before TAVI suggesting a prosthesis-patient mismatch in addition to valve degeneration as the reason for elevated gradients. Since all patients were considered unfit for surgery, the interdisciplinary heart team recommended TAVI to reduce regurgitation.

Further complications included 2 strokes in patients No. 21 and No. 18. In both patients neurological symptoms regressed spontaneously.

In patient No. 18, periprocedural obstruction of the left coronary artery had to be treated with by stent implantation in the left main stem.

All patients were discharged after an average postoperative stay of $10 \pm 8$ days. All-cause mortality after 30 days was $0 \%$.

\section{Discussion}

Trancatheter ViV implantation for failing surgical aortic valves represents an advantageous option for high risk patients. The CoreValve Evolut $\mathrm{R}$ is a new generation valve with approval for $\mathrm{ViV}$ implantation.

Insufficient reduction of the transaortic gradients after TAVI is a major problem for ViV implantations in small surgical valves - especially in patients with prosthesis-patient mismatch [7]. Accordingly, 4 patients in this study with prosthesis-patient mismatch, transaortic gradient could not be improved significantly. In these patients, however, the indication for ViV was a mix of aortic 


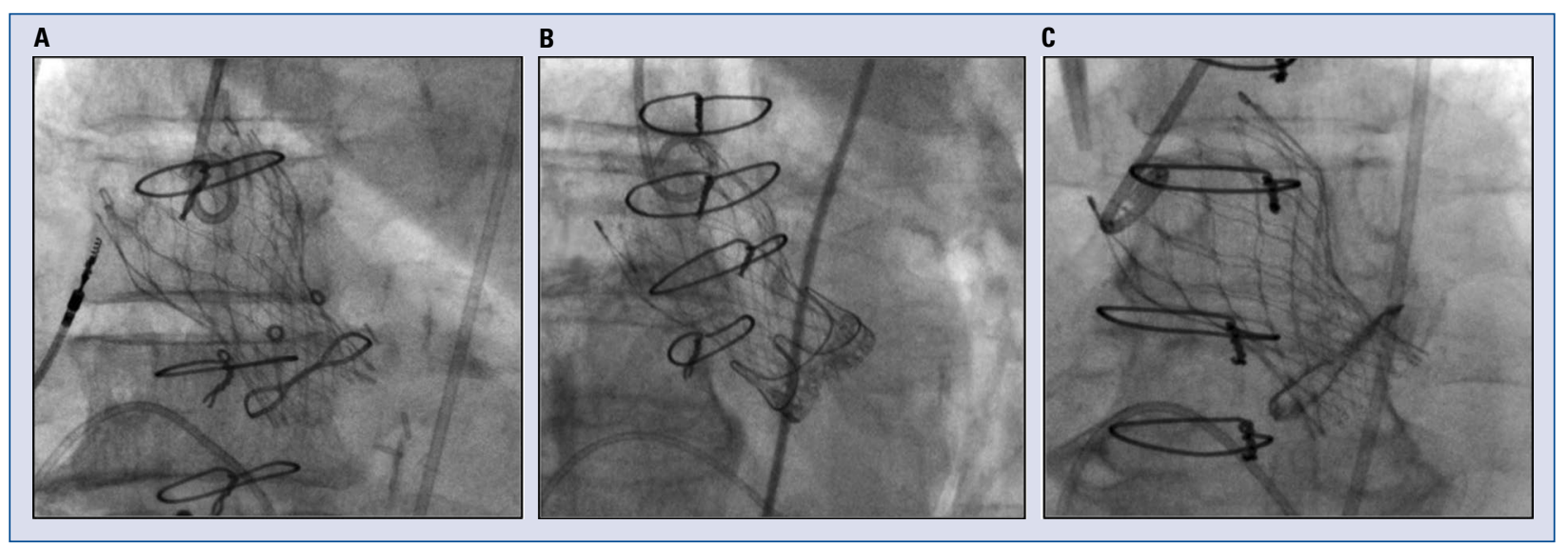

Figure 3. Examples of valve-in-valve implantations in patients with different surgical bioprostheses; A. Hancock; B. Carpentier; C. MitroFlow.

valve disease with predominant aortic regurgitation which was sufficiently reduced. Nevertheless, ViV implantations in patients with suspected prosthesis-patient mismatch should be reserved for patients with very high operative risk. The rate of insufficient reduction of the transaortic gradient was $19 \%$ and therefore comparable with the rate for postprocedural elevated gradients in small valves of $23.4 \%$ for a previous generation of self-expanding valves [8].

Moderate or severe paravalvular regurgitations were not observed in any cases. While clearly limited by the low number of patients included, the present data suggests that the new CoreValve Evolut R is suited for successful interventional treatment of degenerated surgical prosthetic valves. This is an improvement over data for the older generation of self-expanding valves with a rate for at least moderate regurgitation of $8.9 \%$ [8].

Despite the high EuroScore II and STS score no patient died within 30 day follow-up.

According to the available literature, ViV implantations are associated with a lower incidence of relevant conduction disturbances - most likely due to the ability of rigid rings of stented surgical valves which prevent pressure load on the atrioventricular conduction system during TAVI [7]. In 2 patients, complete atrioventicular block occurred after ViV and permanent pacemaker implantation was performed. Notably, a stentless Baxter Prima $25 \mathrm{~mm}$ was the degenerated surgical valve in 1 patient and a very deep implantation of Evolut was necessary to avoid obstruction of the right coronary artery. In the other patient, again a stentless Elan $27 \mathrm{~mm}$ valve was the degenerated surgical valve and a very deep implantation of the Evolut R was necessary to cover paravalvular regurgitation. Both factors likely contributed to heart block in our patients as we have previously shown that deeper implantation is associated with higher risk for permanent pacemaker implantation in patients receiving an Edwards Sapien 3 [9]. The third pacemaker was implanted due to, most likely preexisting, sick sinus syndrome. This rate is comparable to the rate of permanent pacemaker implantation after ViV procedure with previous generations of self-expanding valves [8].

In one other patient, the ViV implantation did not reduce the transaortic gradient. In this patient, a first CoreValve Evolut $\mathrm{R}$ dislocated into the ascending aorta after supraannular implantation. A second valve was placed intraannularly and thereby both replaced the degenerated aortic prosthesis and fixated the first valve. In addition, in this patient the surgical valve was a $19 \mathrm{~mm}$ Carpentier aortic valve. In preoperative computed tomography-scan, an internal diameter of $18 \mathrm{~mm}$ was measured. The combination of a small surgical valve and the necessity of two transfemoral valves probably resulted in an unchanged gradient over the aortic valve. This result is comparable with the literature for the previous generation of self-expanding ViV procedures with a rate which required a second valve of $7.5 \%$ [8].

In general, current literature suggests that an intraanular placement of the valve may result in residual stenosis due to incomplete expansion and leaflet distortion. Accordingly, a supraannular placement of the valve in ViV implantations should be performed to optimize EOA as shown in Figure 3 [10]. 


\section{Conclusions}

It is concluded that the CoreValve Evolut $\mathrm{R}$ is well suited for ViV implantation. In patients with high surgical risk, transcatheter ViV implantation represents a beneficial option with low periprocedural complications. Promising early results should be confirmed by a larger series with long-term follow up.

\section{Conflict of interest: None declared}

\section{References}

1. Potter DD, Sundt TM, Zehr KJ, et al. Operative risk of reoperative aortic valve replacement. J Thorac Cardiovasc Surg. 2005; 129(1): 94-103, doi:10.1016/j.jtcvs.2004.08.023, indexed in Pubmed: 15632830 .

2. Eggebrecht H, Schäfer U, Treede H, et al. Valve-in-valve transcatheter aortic valve implantation for degenerated bioprosthetic heart valves. JACC Cardiovasc Interv. 2011; 4(11): 1218-1227, doi: 10.1016/j.jcin.2011.07.015, indexed in Pubmed: 22115663 .

3. Khawaja MZ, Haworth P, Ghuran A, et al. Transcatheter aortic valve implantation for stenosed and regurgitant aortic valve bioprostheses CoreValve for failed bioprosthetic aortic valve replacements. J Am Coll Cardiol. 2010; 55(2): 97-101, doi: 10.1016/j.jacc.2009.06.060, indexed in Pubmed: 20117377 .
4. Jategaonkar SR, Scholtz W, Horstkotte D, et al. Transfemoral aortic valve-in-valve implantation with the CoreValve Evolut for small degenerated stented bioprosthesis. J Invasive Cardiol. 2014; 26(6): 291-294, indexed in Pubmed: 24907087.

5. Diemert P, Seiffert M, Frerker C, et al. Valve-in-valve implantation of a novel and small self-expandable transcatheter heart valve in degenerated small surgical bioprostheses: the Hamburg experience. Catheter Cardiovasc Interv. 2014; 84(3): 486-493, doi: 10.1002/ccd.25234, indexed in Pubmed: 24753352.

6. Manoharan G, Walton AS, Brecker SJ, et al. Treatment of Symptomatic Severe Aortic Stenosis With a Novel Resheathable Supra-Annular Self-Expanding Transcatheter Aortic Valve System. JACC Cardiovasc Interv. 2015; 8(10): 1359-1367, doi: 10.1016/j. jcin.2015.05.015, indexed in Pubmed: 26315740.

7. Dvir D, Webb J, Brecker S, et al. Transcatheter aortic valve replacement for degenerative bioprosthetic surgical valves: results from the global valve-in-valve registry. Circulation. 2012; 126(19): 2335-2344, doi: 10.1161/CIRCULATIONAHA. 112.104505, indexed in Pubmed: 23052028.

8. Dvir D, Barbanti M, Tan J, et al. Transcatheter aortic valve-invalve implantation for patients with degenerative surgical bioprosthetic valves. Curr Probl Cardiol. 2014; 39(1): 7-27, doi: 10.1016/j. cpcardiol.2013.10.001, indexed in Pubmed: 24331437.

9. Schwerg M, Fulde F, Dreger H, et al. Optimized Implantation Height of the Edwards SAPIEN 3 Valve to Minimize Pacemaker Implantation After TAVI. J Interv Cardiol. 2016; 29(4): 370-374, doi: 10.1111/joic.12302, indexed in Pubmed: 27240558.

10. Simonato M, Azadani AN, Webb J, et al. In vitro evaluation of implantation depth in valve-in-valve using different transcatheter heart valves. EuroIntervention. 2016; 12(7): 909-917, doi: 10.4244/EIJV12I7A149, indexed in Pubmed: 27639744. 